\title{
Academic Department Chair Training: The Why and The How of It
}

\section{Ann F. Lucas}

Fairleigh Dickinson University

Colleges and universities are confronting an unprecedented need for coordinated leadership at every level in the organization. The tensions in higher education that have been triggered by declining enrollments and general financial cut-backs can result in poor faculty morale which can impair teaching effectiveness and increase alienation and fragmentation within an institution. However, these same tensions can serve as a catalyst for creative innovation, program development, and cohesive team work. At such a period in the history of any academic institution, leadership must emerge, not only at the top, but at the departmental level.

The academic department chair occupies a key position as a leader in higher education. Strong leadership skills are required; yet, in most institutions, there is no formal training provided for the chair. As most new chairs attempt to cope with the demanding tasks of their jobs, they discover that the power derived from the position they hold is not enough to accomplish what needs to be done. Departmental goals can be achieved only by working effectively through others, and this requires the exercise of strong leadership skills. Most chairs then rely on whatever level of interpersonal skills they possess, recall the behavior of good and bad role models, and depend heavily on trial and error.

Since the development of leadership skills and a knowledge 
of management science are too important to an organization to permit their occurrence in a haphazard fashion, colleges and universities must plan for systematic leadership development of academic chairs.

Little academic chair training has occurred in higher education. ${ }^{1}$ In part, this is because chairs and faculty resist the idea that the department chair is a manager, believing that managers are part of industry, not academe. Yet academic chairs do manage and are responsible for the work of the people in their organizational units. Chairs do serve as a necessary conduit, representing faculty to the dean and sharing information about organizational goals to the faculty. The significant difference, however, is that the academic chair often has little of the formal power of a manager in industry. Motivating a difficult tenured faculty member is perceived by many chairs as close to impossible. The frequent lament is, "We have neither carrots nor sticks. How can we be effective leaders?"

\section{Academic Chairs Are Not Trained For Their Leadership Role}

Such comments reflect a lack of exposure to formal leadership training. Most academic chairs are not at all clear about what motivates faculty or what role they can play in that process. Unaware that a whole body of literature has already addressed these problems, they wrestle alone, feeling that to admit difficulty in solving such problems might reflect on their adequacy as department leaders. While chairs may sometimes complain to department members that they are constantly dealing with administrative minutiae, most chairs do enjoy the work and would make the same choice of becoming chair again. (Tucker, 1984). However, it is part of the academic culture that they must protest to others that they have taken the job because "someone has to do it."

A faculty member becomes an academic chair by being elected by members of the department, through appointment by the dean, through an established system of rotation, or some combination of these factors. The person is usually selected because he is respected, is felt to be knowledgeable about the discipline and the department, and is perceived to be fair in dealing with others. 
When invited to become chair, a faculty member may accept for a number of reasons. Tucker (1984) includes in his list: personal satisfaction in helping others develop professionally, a chance to build effective academic programs, the challenge of leadership, defending the interests of the department, access to deans and vice-presidents, and status and prestige. Demonstrated ability in performing leadership functions is seldom a determining characteristic either for those who make the selection or for those who accept the invitation to become academic chairs.

Thus the dilemma is created. A faculty member is selected to become chair. He or she accepts the challenge. But the knowledge and skills that can make a leader effective must somehow be learned through trial and error. Most departments will permit a "honeymoon" period during which a chair may have an opportunity to solve problems and be innovative. However, by the beginning of the second semester, the chair's personal power will be reduced if he or she is judged to be ineffective in defending the department's causes or if perceived to be unfair, either in allocation of resources or in the role as final arbiter of faculty's teaching schedules. When this occurs, a department may accomplish little for the duration of the chair's term of office, remaining in a kind of holding pattern until the next chair takes office.

The purpose of the present paper is to discuss: (a) the kinds of training that a chair would find most useful in maximizing his effectiveness as leader of the department, and (b) the steps that a change agent can take in order to bring about effective leadership training for the academic chair.

\section{NECESSARY INGREDIENTS FOR ACADEMIC CHAIR TRAINING}

Effective leadership training for academic chairs must include two basic components: (a) learning some key elements in the body of literature that deals with leadership and management functions, and (b) an opportunity to identify and practice leadership skills. The two essential aspects of the subject of leadership are: interpersonal effectiveness and small group leadership. These topics include the fields of self-awareness, communication, feedback, leadership style, power and influence, motivation, performance counseling, conducting effective 
meetings, problem solving and decision making, negotiation, conflict resolution, and team building.

The Director and a staff member ${ }^{2}$ of the Office of Professional and Organizational Development (POD) at Fairleigh Dickinson University have developed and used a unique model in our Leadership Development Program for academic chairs (Lucas, 1986). This model provides intensive work with chairs in one college at a time for an entire semester. The format includes ten three-hour sessions which were divided into minilectures of 20 to 30 minutes, a discussion of handouts which had been distributed the week before, role play (behavior rehearsal) of scenarios of actual problems academic chairs were currently wrestling with, feedback from participants and observers of that role play, a discussion of what had been learned or back-home application of learnings, and an evaluation of the session. (See Appendix for a list of topics addressed in our program.)

Such a format permits learning of information and skills which are directly related to the problems that currently engage the attention of the academic chairs. The learning experience is validated for the participants because it is focused on issues that are of immediate concern to them.

The value of a lengthy (one semester), intensive model for learning is that it provides the necessary time for trust to be built in the facilitator(s) and in the group members. There is also the opportunity to confront the natural resistance and skepticism, and to continue with the group long enough so that they can experience the benefits of leaving a session with several alternative solutions to a problem with which they have been struggling. Even more importantly, they will have practiced the skills that are needed, e.g., in dealing with a difficult faculty member or resolving a conflict in the department.

The model used in our Leadership, Development Program has created a peer support group that has enabled chairs to reach out to each other to discuss new problems as they occur. As part of the follow-up work, a number of chairs have also requested help from the POD Office with particular departmental problems. In two conflict-ridden departments, for example, we have worked closely with the individual chairs to do some team building, keeping chairs in those departments apprised constantly of what we are doing, asking for their advice and 
feedback on what would and would not be likely to work given the culture of those departments.

\section{SEQUENCE OF STEPS NECESSARY FOR EFFECTIVE ACADEMIC CHAIR TRAINING}

In order to conduct leadership training in an institution where such programs have not previously been sponsored, some important steps must be undertaken:

1. An awareness of the need for leadership training must be developed. One approach that is almost certain to work is to invite an "expert" in from outside to speak to chairs and faculty members about leadership in the academic department. Then arrange for chairs in small groups to identify their own needs in leadership development. Summarize the results and provide this information to the conference participants.

2. You, as a change agent in your college or university, can then share your findings on the leadership needs chairs have identified with the appropriate academic officer, i.e., dean or VPAA. All training or change efforts have the greatest chance of being successful if there is support from top administration.

3. Conduct a needs analysis for one segment of your total system, i.e., a college if you are in a university. Interview and secure the support of the dean of that college. Find out what the dean perceives to be the strengths and the needs of the college. Then ask the dean to identify several chairs you can also interview. These chairs should represent a diversity of points of view so that a range of opinions can be tapped. Conduct what is called "sensing" interviews (Jones, 1973) with each of these individuals. Such an interview has as its goals: (a) generating useful data to form the basis for an organizational diagnosis, (b) gaining an in-depth understanding of data that have been obtained, and (c) developing ownership in the process of creating changes in the system.

4. Based on the data you have now collected, formulate some hypotheses about the leadership needs of the college. Develop a list of program objectives and submit it to the dean for additions, deletions, and approval. Let the dean know that you will be reporting back at each stage of the project so that there will be no surprises. At the same time, however, it is essential that confidentiality be observed at every step along 
the way. Any information picked up in interviews should not be communicated to any other member of the college.

5. Have the dean announce the fact that a leadership program for chairs will take place. Let him or her handle all of the communication about the program. If the dean and chairs work reasonably well together, it would be useful to include the dean in the leadership development sessions. One value of this strategy is that an opportunity can be provided for chairs and dean to discuss mutual expectations of the role of the chair. Further, communication can be opened up between the two levels of chair and dean, so they can problem-solve together. It is extremely important to discuss ahead of time what role the dean will play in the leadership development program. What is most helpful is if the dean is supportive of the chairs and encourages them, through example, to be open and nondefensive.

6. Utilize all of the behaviors that are normally required of a good group facilitator, i.e., create a supportive climate in which chairs can feel free to experiment with new behaviors, build trust, provide positive feedback to participants, establish positive norms for the group, and accept all feedback from the group without being defensive.

7. Let the group know that you are available for individual consultation with any specific departmental problems and treat all of this with strict confidentiality.

8. Evaluate the program, both at the end of each session and also at the end of the entire program.

9. Provide for follow-up work with the group. Participants might plan a half-day session in which they can report progress on how they have tried to solve problems since the group ended. Other chairs can give feedback and recommend alternate strategies. The goal is to encourage chairs to become a support network for each other.

You as the change agent can also provide follow-up work with individual departments; e.g., engaging in process consultation for departmental or committee meetings; third party arbitration between a chair and a difficult faculty member; strategizing with a chair on handling some departmental problem; or assisting chairs in goal setting, bringing about change, and team building. 


\section{ADVANTAGES OF THE PROPOSED MODEL}

The complaint one hears most frequently about management or leadership effectiveness programs is that the conference effects don't last. Participants feel euphoric at the end of the program and are convinced that they have learned a number of useful strategies for handling problems. However, when managers return to the office, they face the same uphill struggle in getting things done because the same difficult people and the same system problems continue to be present. Hopes are dashed, and leaders feel even more discouraged.

The value of this prolonged, intensive model is that once a level of trust that allows problems to surface has been established, the problems can continue to be worked on as the group meets over time. Also, because the change agent or group facilitator continues to be available on-site after the program has been completed, individual chairs have access to ongoing, confidential help with problems from someone whose competence they respect. Thus, unlike other approaches, this model strongly increases the probability of making a significant impact on the organization.

\section{APPENDIX}

Session Topics:

1. The roles and responsibilities of a department chair. The conflicting roles that the chair must assume in carrying out responsibilities to both administration, faculty, and students were explored. The knowledge and skills necessary to perform these functions were identified. Program objectives were presented so that chairs could add their own goals.

2. Leadership and communication. A brief review of leadership research, the task and relationship aspects of the role, and the way in which these functions apply in the department were covered. A discussion of the key elements in the communication process and an opportunity to role-play interactions between a chair and a faculty member were provided.

3. Motivating faculty to assume greater responsibility in departmental functioning. Creating a climate in which faculty can achieve their own professional goals by working towards organizational goals is the key to successful faculty motivation. Active listening with faculty and providing constructive feedback were practiced. 
4. Resolving conflict through problem solving. The value of encouraging conflict in the department as a basis for developing a broad range of perspectives for problem solving was emphasized. A problem solving model was discussed, and the generation of implementation steps was role-played using situations developed by participants.

5. Developing skills in performance counseling. Handling performance counseling with faculty, with an emphasis on establishing goals and concrete action steps, was discussed. Several alternative approaches to an actual problem with a difficult faculty member were role-played, and feedback given to participants.

6. The department chair as team leader. The properties of effective task groups were identified. Through simulations of actual departmental problems, participants practiced and observed appropriate task and maintenance functions in groups.

7. Decision making in the department. The different methods of decision making were discussed, i.e., unilateral, delegation, consultation, and consensus, along with the anticipated effects of each approach. A task group meeting was simulated, and the effects of the decision making style adopted were analyzed.

8. Bringing about change in the department. The emergence of norms in our own work group and how they affect behavior were discussed. Departmental norms were identified. How negative norms in the department can be changed was demonstrated through a structured experience in which a chair confronted a negative norm, i.e., avoiding conflict by not verbalizing disagreement.

9. Managing conflict in the department. The varieties of ways in which conflict can be useful were discussed. A film clip on third-party intervention was shown. Role-plays of a chair dealing with a faculty member who does not make any contribution to the department, aside from teaching his class, demonstrated several approaches to handling conflicts with individual faculty.

10. Goal setting, evaluation of training, and follow-up program. Participants determined their needs as a group for further leadership development, setting up plans for follow-up work. Availability of POD staff for further individual work in departments was emphasized. The training program was evaluated both orally and in written form.

\section{REFERENCES}

Blom, D.I. (1985). Management training for university administrators: The University of Tennessee experience. Journal of Staff, Program, and Organization Development, 3(2), 58-62.

Cashin, W.E. (1984). Proceedings of Academic Chairpersons: Administrative Responsibilities, 13. Manhattan, Kansas: Kansas State University, Center for Faculty Evaluation and Development, Division of Continuing Education.

Cashin, W.E. (1985). Proceedings of Academic Chairpersons: Leadership and Management, 17. Manhattan, Kansas: Kansas State University, 
Center for Faculty Evaluation and Development, Division of Continuing Education.

Lucas, A.F. (1986). Effective department chair training on a low cost budget. Journal of Staff, Program, and Organization Development, 4(2), 33-36.

Jones, J.E. (1973). The sensing interview. In J.W. Pfeiffer \& J.E. Jones (Eds.), The 1973 annual handbook for group facilitators. San Diego, CA: University Associates. 213-224.

Tucker, Allan. (1984). Chairing the academic department (2nd ed.). New York: Macmillan.

\section{NOTES}

1. Notable exceptions are the American Council on Education Leadership Development Workshops, The University of Tennessee basic management training program (Blom, 1985), Michigan State University programs on performance evaluation, and Kansas State University conferences on Academic Chairpersons (Cashin, 1984-1985).

2. The author is pleased to acknowledge the work of Dr. Martin Birnbaum who collaborated fully in all phases of the FDU Leadership Development Program. 